\title{
Guards in Action: First-Order SCA Secure Implementations of Ketje without Additional Randomness
}

\author{
Victor Arribas \\ KU Leuven, imec-COSIC, Belgium \\ victor.arribas@esat.kuleuven.be
}

\author{
Svetla Nikova \\ KU Leuven, imec-COSIC, Belgium \\ svetla.nikova@esat.kuleuven.be
}

\author{
Vincent Rijmen \\ KU Leuven, imec-COSIC, Belgium \\ vincent.rijmen@esat.kuleuven.be
}

\begin{abstract}
Recently the CAESAR competition has announced several finalists among the submitted authenticated encryption algorithms, after an open selection process during the last five years. Applications using these algorithms are rapidly increasing today. Devices implementing these applications are enormously susceptible to physical attacks, which are able to retrieve secret data through side-channel information such as power consumption or the electromagnetic radiations. In this work, we present a Side-Channel Analysis resistant hardware implementation of the whole family of authenticated encryption schemes KETJE. By changing just one parameter, any of the KETJE designs can be obtained, and tailored for different applications, either lightweight or high throughput.

We introduce a new protected KECCAK implementation, as well as unprotected and protected KETJE implementations, which allow both encryption and decryption modes in the same module. In order to secure these implementations we make use of the masking scheme known as Threshold Implementations and complement it with the technique of "Changing of the Guards", achieving a first-order Side-Channel Analysis protected implementation with zero extra randomness needed. This way, no dedicated PRNG needs to be additionally implemented, avoiding issues such as the security of the PRNG itself or the quality of the randomness. We elaborate on the importance of the input dependencies and how the addition of linear blocks could thwart the security provided by a correctly protected module. This is of special importance when extending KECCAK to implement KETJE. Furthermore, we provide an empirical evaluation of both KECCAK and KETJE implementations, demonstrating how with our methodology we can construct a secure KETJE based on a previously secured KECCAK.
\end{abstract}

Index Terms-Authenticated Encryption, KETJE, SHA-3, SideChannel Analysis, Threshold Implementations, Changing of the guards.

\section{INTRODUCTION}

The use of IoT devices is growing enormously, with predictions of an increase of $250 \%$ in their use in the next three years. This makes hardware security an important issue, needed to protect devices implementing cryptographic algorithms. Several attacks have proliferated in the literature in the past few years, Side-Channel Analysis (SCA) being one of the most significant given its reduced cost and its ease of use. They exploit computation time, power consumption or electromagnetic radiation to extract sensitive information such as cryptographic keys. A distinct and very powerful SCA is Differential Power Analysis (DPA) [1].
Multiple techniques have been proposed to secure the hardware, as can be hiding or masking [2] among others. Masking has caught most of the attention in the literature, with several masking schemes proposed [3]-[8]. The aim of masking is to randomize the intermediate variables, making the power consumption independent of their value. The first works of Ishai et al. [3] and Trichina [4] were proven flawed, since they did not provide security in the presence of glitches. The work of Nikova et al. [5], known as Threshold Implementations (TI), was the first to provide first-order SCA security in the presence of glitches and later extended to higher-order by Bilgin et al. [6]. Reparaz et al. [7], in Consolidated Masking Schemes (CMS), reduce the area overload to achieve the same order of security at the cost of introducing extra randomness, and provide security against multivariate attacks (higher-order attacks where wires in different time frames are probed). Subsequently, Gross et al. [8], in Domain Oriented Masking (DOM), propose a reduction in the randomness with respect to CMS.

Our contribution. In this work we present a new protected KECCAK implementation with new trade-offs: slightly bigger area compared to the smallest state-of-the-art parallel implementations, but two or three times lower latency. Additionally, we introduce the implementation of the whole KETJE family, both unprotected and first-order SCA protected with zero extra randomness added. Furthermore, we extend these implementations to allow encryption and decryption in the same module, both protected and unprotected. We deploy the firstorder protected KECCAK in FPGA and test it, proving the implementation secure for up to 100 million traces. We expand this implementation to get the secure KETJE implementation that inherits the previously proven security, since the expansion only involves linear operations and we use a TI sharing scheme.

We further extend this work with a discussion on the importance of the input dependencies in masked implementations and how failing to satisfy this requirement can endanger their security. In [9], we claimed that the KETJE implementation was secure by just evaluating the underlying permutation. This assumption was justified given that only linear operations were utilized on top of the secure KECCAK to design KETJE, with TI as countermeasure. To verify the correctness of our approach, in this work we perform the empirical evaluation on the protected KETJE encryption implementation. We explain in detail the 
points of analysis for both evaluations. The results obtained match our expectations, i.e., we find no leakage in the protected KETJE. Additionally, we find that KETJE provides certain SCA security, up until a limited number of computations reusing the same key.

We begin by summarizing the specifications of KETJE in Section II, then we discuss important concepts and methodologies applied to secure our implementations in Section III. Details of the protected implementations are given in Section IV, followed by the results and evaluation details in Sections $\mathrm{V}$ and VI respectively.

\section{KetJE}

KetJE [10] is a CAESAR (Competition for Authenticated Encryption: Security, Applicability and Robustness) third round candidate. Although recent updates on the CAESAR competition do not include KETJE as a finalist, we still believe it is a highly valuable algorithm. It is based on the SHA3 standard and it is very suitable for side-channel resistant hardware implementations. KETJE is a set of four authentication encryption schemes that allow messages with associated data. They target memory-constrained devices and strongly rely on nonce uniqueness for security. Ketje is built following a layered design, where KECCAK [11] is the underlying permutation, called by the construction MONKEYDUPLEX, which at the same time is instantiated by the mode MONKEYWRAP.

\section{A. KECCAK}

KECCAK is a family of hash functions that were standardized by NIST, becoming SHA-3. It runs the permutation KECCAK$f[b]$ where $b \in\{25,50,100,200,400,800,1600\}$ defines different state sizes. KETJE uses a round reduced permutation KECCAK- $p\left[n_{r}, b\right]$, which runs the last $n_{r}$ rounds of KECCAK- $f$ $[b]$. KECCAK works on a three dimensional state (Fig. 1), where a round consists on five operations: $R=\iota \circ \chi \circ \pi \circ \rho \circ \theta$. We refer to [11] for further details on the operations.

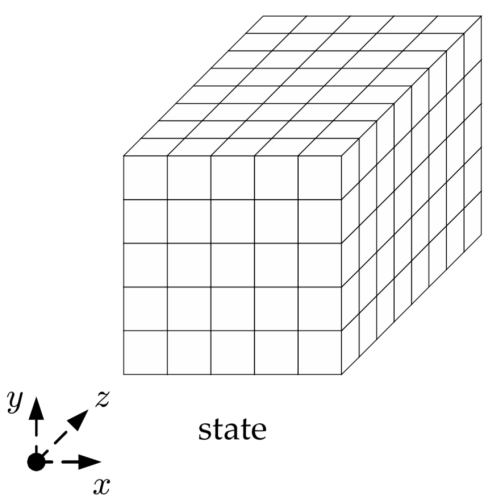

Fig. 1: Three-dimensional KECCAK state [11]

\section{B. MONKeyDuplex}

MONKEYDUPLEX calls a round-reduced version of KECCAK- $f$ where different types of calls are supported invoking different numbers of rounds. The performance of the functions can be optimized by reducing the number of rounds, at the expense of requiring nonce uniqueness for the scheme to provide security against key retrieval. MONKEYDUPLEX follows the structure of a duplex construction, where there is an absorption phase and a squeezing phase alternating. The absorption phase introduces new bits to the state, while the squeezing phase extracts bits from the state. Fig. 2 depicts this idea. The function takes as input (absorbs) a binary string of any length $\sigma$ and returns (squeezes) a binary string of the requested length $Z$.
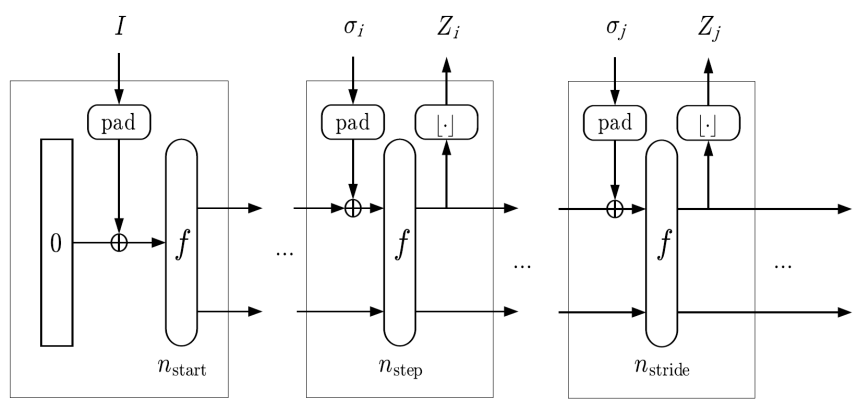

Fig. 2: The MonkeyDupleX construction [10]

Four parameters $\left(r, n_{\text {start }}, n_{\text {step }}, n_{\text {stride }}\right)$ determine the efficiency and security strength of the module. Two types of calls are supported by MONKEYDUPLEX:

- D.start: at the beginning the string $I=K \| N$ that concatenates the Key $(K)$ and the Nonce $(N)$ is introduced in the function after a simple padding is applied, initializing the state. Then, the function $f$ runs for $n_{\text {start }}$ rounds, $f\left[n_{\text {start }}\right]$.

- D.step or D.stride: the construction absorbs a data block $\sigma$, extended with a multi-rate padding until the string length is equal to the rate $r$. Then, either $f\left[n_{\text {step }}\right]$ or $f\left[n_{\text {stride }}\right]$ is applied to the state $f$, and afterwards part of this state is extracted $(Z)$.

This construction can be used for mainly three different applications: first, the simplest one, as a stream cipher; second, as a reseedable (fresh seed every block) pseudorandom bit sequence generator; third, and the most relevant one, as authenticated encryption.

Finally, it is important to stress that both uniqueness and secrecy of $I$ are crucial to comply with the security claims. In addition to this, multiple instances of MONKEYDUPLEX with the same input $I$ are not allowed, since, otherwise, an attacker could retrieve the full state easily by observing differences in the output.

\section{MONKEYWraP}

The authentication process takes as input a header $A$ or Associated Data, a data body $B$ or Plaintext, and returns a Ciphertext $C$ and a Tag $T$. The encryption process is known as wrapping and then the decryption process is known as unwrapping. The functionality of the encryption is outlined below and illustrated in Fig. 3:

1) The state is initialized with Key and Nonce executing $D \cdot \operatorname{start}(\operatorname{keypack}(K,|K|+16)|| N)$ where the keypack is a way of encoding the secret key given a string input. 


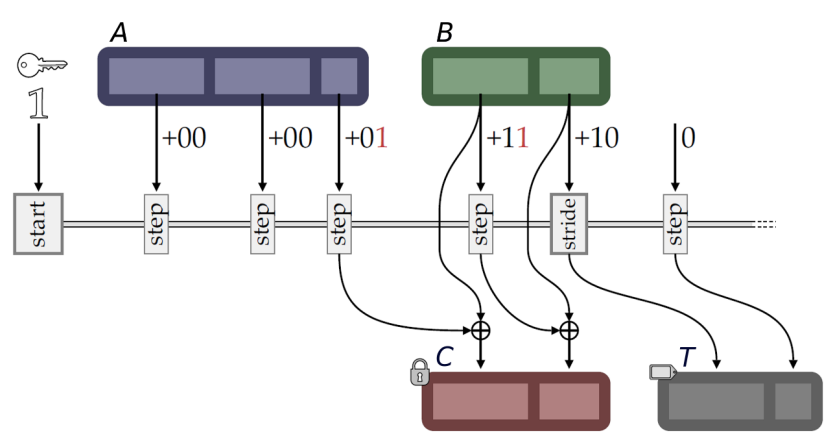

Fig. 3: The MonkeyWraP construction [10]

2) The associated data is processed block by block $\left(A_{i}\right)$ by executing $D \cdot \operatorname{step}\left(A_{i} \| 00\right)$, and $D \cdot \operatorname{step}\left(A_{i} \| 01\right)$ for the last block of $A$. The block size $\left(\left|A_{i}\right|\right)$ of the mode is given by $\rho$. The last output is XORed with the first plaintext block to get the first ciphertext block.

3) The plaintext is similarly processed with $D \cdot \operatorname{step}\left(B_{i} \| 11\right)$ and the output of every step is XORed with the next block of the plaintext to get a ciphertext block. The last block of $B$ is treated with $D \cdot \operatorname{step}\left(B_{i} \| 10\right)$ to produce the first tag block.

4) Subsequent tag blocks are produced by executing $D$.step $(0)$ until the required $T$ length is achieved.

The decryption is essentially identical to the encryption except for few differences: the scheme receives as input $A$, $C$ and $T$, where the same procedure is followed, using the ciphertext data instead of the plaintext. The new tag $T^{\prime}$ is calculated out of the plaintext blocks decrypted, and finally the entire plaintext $B$ is output only if $T=T^{\prime}$.

MONKEYWRAP supports sessions, allowing the encryption of several messages (with the respective associated data) with the same input $I$. The state is never reset or reinitialized during the same session, so that the function keeps running with the previously updated state. This means that intermediate tags are produced at the end of every encryption, authenticating the current encryption together with the previous ones. Thus, a sequence of messages can be authenticated, rather than a single one. This offers an easy way of sending successive commands while preventing the interference of an attacker.

The KETJE specifications allow four different authenticated encryption schemes, namely: KetJe JR, KetJe SR, KetJe MinOR and KetJe MAJOR. They are characterized by the underlying permutation and their block size $(\rho)$. They can be used in either lightweight applications or higher-throughput implementations. Tab. I summarizes the four different possibilities.

Important features of the KETJE schemes to take into account:

- As already mentioned above, all but KetJe MAJOR are lightweight.

- The greater block size of KetJe Minor and KetJe MAJOR allows the output of 128-bit tags without extra costs.
TABLE I: Four KeTJE authenticated encryption schemes [10]

\begin{tabular}{lccc}
\hline Name & $f$ & $\rho$ & Main use \\
\hline KETJE JR & KECCAK- $p^{*}[200]$ & 16 & lightweight \\
KETJE SR & KECCAK- $p^{*}[400]$ & 32 & lightweight \\
KETJE MINOR & KECCAK- $p^{*}[800]$ & 128 & lightweight \\
KETJE MAJOR & KECCAK- $p^{*[1600]}$ & 256 & high performance \\
\hline
\end{tabular}

- They are very well suitable for side-channel countermeasures and a perfect fit for secure messaging with secured chips, for instance, smart cards.

\section{SECURING THE HARDWARE}

Hereunder we provide an overview of the concepts needed and used throughout the paper to secure a hardware design, namely: the adversary model, where the adversary capabilities are introduced; Threshold Implementations, where the key concepts to correctly implement this sharing scheme are specified; Input Dependencies, where the importance of this concept in securing hardware implementations is addressed, and finally, the technique known as "Changing of the Guards" to complement the sharing methodology used.

\section{A. Adversary model}

Along this work we consider the model proposed in [3] known as the $d$-Probing Model. A single probe that monitors the power consumption (or the electromagnetic radiance) of a single wire. The adversary gains information of all the intermediate values of the wire from the last register to the probe. Similarly, an adversary probing $d$ wires acquires the knowledge of every intermediate value of all $d$ wires. A $d$ th-order security scheme provides security against up to $d$ th-order attacks.

\section{B. Threshold Implementations}

Threshold Implementations (TI) was first introduced by Nikova et al. [5] for first-order security, and broadened further to higher-order by Bilgin et al. [6]. Like multiple other masking schemes, TI is based on secret sharing, where each sensitive, i.e. key dependent, data element $x$ is divided into $s$ pieces $\left(\mathbf{x}=\left(x_{1}, \ldots, x_{s}\right)\right)$, such that $x=x_{1} \perp \ldots \perp x_{s}$. We consider Boolean masking, where $\perp$ is exclusive addition $\oplus$, and all $s$ shares are needed to derive $x$. The aim of this methodology is to provide formal methods to correctly randomize the intermediate variables and hence achieve security in hardware implementations. TI imposes three mandatory properties for a design to be secure:

- Correctness: a shared function $\mathbf{f}$ such that $f_{i}(\mathbf{x})=Y_{i}$, where $i=1, \ldots, s$, is correctly shared if $\sum Y_{i}=Y=$ $f(x)$.

- Non-completeness: a shared function $\mathbf{f}$ is $d^{\text {th }}$-order noncomplete if any combination of up to $d$ component functions $f_{i}$ is independent of at least one input share. Here $d$ is the degree of security.

- Uniformity: the outputs of a shared function have to conform a uniform distribution. Note that given a uniform 
sharing, it is sufficient to preserve the uniformity across operations to achieve univariate security (together with non-completeness).

TI provides security even when the gates' behavior is nonideal, that is, any gate can glitch depending on prior inputs of the same clock cycle before stabilizing without giving any advantage to an attacker thanks to the non-completeness property.

The number of shares $s$ define the degree of security. The lower bounds for the number of input and output shares are given in [6], in function of $d$ and the algebraic degree of the function $t$, following the expressions:

$$
\begin{gathered}
s_{\text {in }} \geq t d+1 \\
s_{\text {out }} \geq\left(\begin{array}{c}
s_{\text {in }} \\
t
\end{array}\right) .
\end{gathered}
$$

We provide an example of how to protect a simple AND/XOR gate $(z=w \oplus x y)$ in Eq. 2 , where $\left(d, t, s_{\text {in }}, s_{\text {out }}\right)=$ $(1,1,3,3)$ with TI:

$$
\begin{aligned}
& Z_{1}=w_{1} \oplus x_{1} y_{1} \oplus x_{1} y_{2} \oplus x_{2} y_{1}, \\
& Z_{2}=w_{2} \oplus x_{2} y_{2} \oplus x_{2} y_{3} \oplus x_{3} y_{2}, \\
& Z_{3}=w_{3} \oplus x_{3} y_{3} \oplus x_{1} y_{3} \oplus x_{3} y_{1} .
\end{aligned}
$$

We denote an input $x$ with lower case letters, an output $Z$ with upper case letters, and we refer to a specific bit of an S-Box with index $b$ as $x[b]$.

\section{Input dependencies}

CMS [7] and DOM [8] are two hardware masking schemes derived from TI. They both aim to reduce the number of shares while maintaining the same degree of security. To achieve this, apart from the need of extra fresh randomness, a new condition must be satisfied, i.e., the inputs have to be independent of each other. To satisfy this condition in every operation is not trivial, since it is difficult to track the different dependencies among every variable of the system. Failing to provide independent inputs leads to fail the non-completeness property, and hence the security of the design.

To illustrate the importance of these input dependencies, we present a very simple example where security is broken due to dependent inputs when a CMS/DOM-like sharing scheme is used. For the sake of clarity, we also provide the secure case in which the inputs are independent. The example uses two shares to secure against first-order analysis. Fig. 4a depicts the example circuit $z=y \cdot f(x)$, and Fig. $4 \mathrm{~b}$ the circuit $z=x \cdot f(x)$. We choose $f$ to be a simple left shift, $f(x)=x \ll 1$, but any linear function would serve.

Eqs. 3 and 4 present in detail the shared operations for the examples in Fig. 4 respectively.

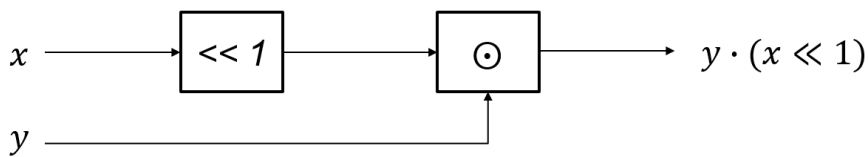

(a) Independent inputs operation

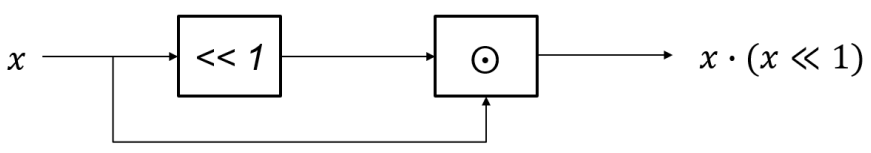

(b) Dependent inputs operation

Fig. 4: Independent and dependent inputs CMS/DOM sharing example

$$
\begin{aligned}
& \mathbf{y}=\left(y_{1}, y_{2}\right) \\
& \mathbf{x} \ll 1=\left(x_{1} \ll 1, x_{2} \ll 1\right) \\
& \mathbf{y} \odot(\mathbf{x} \ll 1)=\left\{\begin{array}{l}
y_{1} \cdot\left(x_{1} \ll 1\right) \\
y_{1} \cdot\left(x_{2} \ll 1\right) \\
y_{2} \cdot\left(x_{1} \ll 1\right) \\
y_{2} \cdot\left(x_{2} \ll 1\right)
\end{array}\right. \\
& \mathbf{x}=\left(x_{1}, x_{2}\right) \\
& \mathbf{x} \ll 1=\left(x_{1} \ll 1, x_{2} \ll 1\right) \\
& \mathbf{x} \odot(\mathbf{x} \ll 1)=\left\{\begin{array}{l}
x_{1} \cdot\left(x_{1} \ll 1\right) \\
x_{1} \cdot\left(x_{2} \ll 1\right) \\
x_{2} \cdot\left(x_{1} \ll 1\right) \\
x_{2} \cdot\left(x_{2} \ll 1\right)
\end{array}\right.
\end{aligned}
$$

Since the multiplication is a non-linear operation, in the computation there appear four cross terms that would later be compressed back to two shares. We stop our computations at this stage, as we observe that in Eq. 4 there is a noncompleteness violation in two of the cross terms, where all shares of $x$ are being used together. This failure appears given that the inputs fed into the multiplier are not independent. In Eq. 3, where the inputs are independent, even though two different shares are used in an operation, these shares are not of the same variable so no secret data can be reconstructed.

TI prevents this from happening, given that there is always at least one share missing for every variable (see Eq. 2). This justifies the assumption that only by adding linear operations into the system, this would remain secure and no leakage would be introduced.

As shown in [12], this concept is of particular importance in KECCAK, given the intricate shape of the initial linear permutation $\theta$. This work points out a problem in a DOMKECCAK implementation, due to dependent inputs. Hence, we decided to use TI to secure our implementations in order to avoid these problems. 


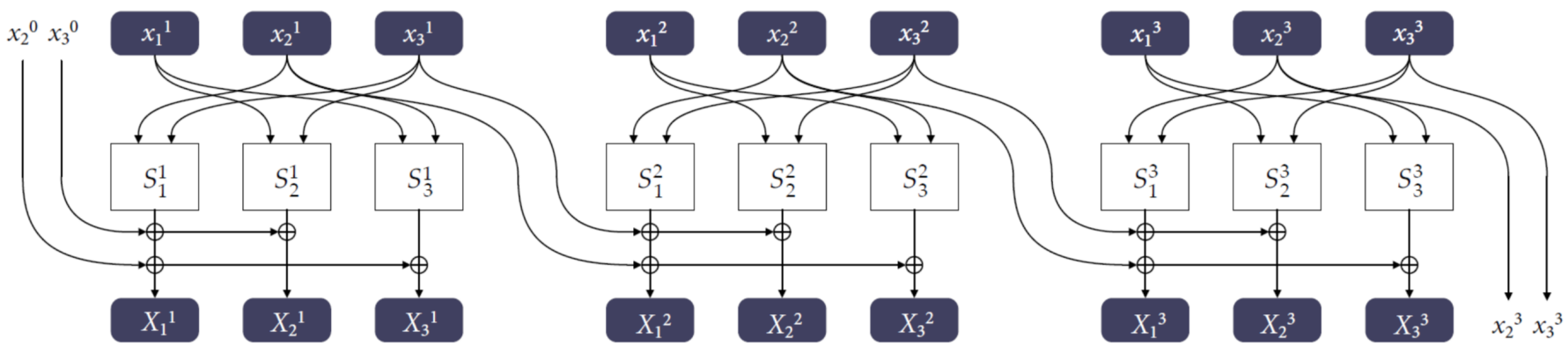

Fig. 5: "Changing of the Guards" structure for three adjacent S-Boxes from a three shares secure implementation [13]

\section{Changing of the Guards}

In [13] Daemen proposes a method to achieve uniformity in a shared S-Box by just appending few dummy bits to the states, also known as "guards". This method uses these first bits to randomize the first S-Box $x^{1}$, then a few of the output bits of this S-Box are similarly used to randomize the second S-Box $x^{2}$, and so on until the last S-Box $x^{j}$. The last output bits will be the new "guards" of the next round. By feedforwarding these bits and the small extension of the shares, uniformity in the whole S-Box layer is achieved instead of making every single shared S-Box uniform. Fig. 5 illustrates a simple example of a sequence of three shared S-Boxes with three shares each.

This technique has been used previously to protect ASCON and KeYAC [14], and also in a first-order secure AES [15].

\section{IMPLEMENTATIONS}

In this section we provide the details of the whole KETJE implementation. The design follows the layered structure given in the specifications of Sect. II. We start from the very bottom, with the underlaying permutation KECCAK which is implemented in a round-based manner. We continue with the MonKeyDuPlex block that calls the permutation. Finally, we implement MONKEYWraP, which instantiates MONKEYDUPLEX, for encryption, and further extended to support encryption/decryption together. The implementation is made generic, where, by just varying the width of the permutation (b), the four instances of KETJE can be obtained.

\section{A. KECCAK-p*}

a) Twisted permutations: In KeTJE v2, the underlying permutation is modified to the twisted permutation KECCAK$p^{*}=\pi \circ \operatorname{KECCAK-~} p \circ \pi^{-1}$. The purpose of this updated version of the underlying permutation is to more effectively re-order the state bits. Since our implementation is round-based, the update and implementation of the twisted permutation is trivial, simply by instantiating $\pi$ and $\pi^{-1}$ at the end and at the beginning of the round function respectively. Fig. 6 illustrates the round function of the twisted permutation.

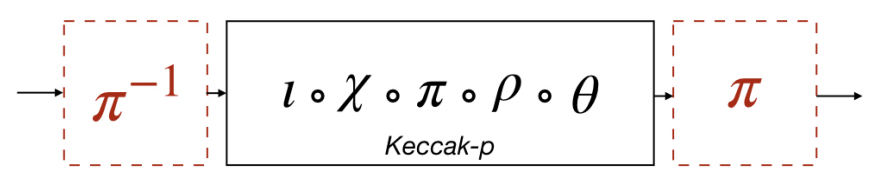

Fig. 6: KЕССАК- $p^{*}$ round function extending the original KЕСCAK- $p$

b) Design choice: There are several protected implementations of KECCAK proposed in the literature [16]-[18]. The first two use TI to secure their implementations: the first one was shown to use a non-uniform sharing, which was fixed by Bilgin $e t a l$. in the second one by using a four shares scheme instead of three to achieve uniformity. Gross et al., in [18], propose several serial and parallel implementations using DOM to secure their designs. KETJE is designed to operate best with a parallel implementation of KECCAK to get the best trade-off between throughput and area. KETJE makes one call to the $D$.step function for every block in the Associated Data and in the Plaintext. To avoid excessive increase in latency, we seek to compute D.step within one clock cycle. We discard implementations using DOM since a single round of KECCAK would need two or three cycles to be computed in the parallel implementations. We then go with TI to get the best latency, and we apply the "Changing of the Guards" method explained before, to get a uniform sharing with three shares. With this method we are able to optimize area with respect to the fourshares version, and no extra randomness is needed. Thus, no dedicated PRNG is needed to feed this module.

Securing linear operations in TI is trivial, where just mere repetition is needed to get correctness. Hence, we focus on how to secure the non-linear operation $\chi$ and how the guards are implemented. Eq. 5 shows the equations followed in our first-order implementation with three shares, similarly given in [13].

It is difficult to compare our implementations area-wise with previous works given that different synthesis libraries were used. However, latency can be compared precisely. Since no registers are needed to secure the non-linear operation, the corresponding KECCAK- $f$ implementation takes respectively two times and 
For $0 \leq b \leq 2$ :

$X_{1}^{j}[b] \leftarrow x_{3}^{j}[b] \oplus\left(x_{3}^{j}[b+1] \oplus 1\right) x_{3}^{j}[b+2] \oplus x_{3}^{j}[b+1] x_{1}^{j}[b+2] \oplus x_{1}^{j}[b+1] x_{3}^{j}[b+2]$ for $j>0$
$X_{2}^{j}[b] \leftarrow x_{1}^{j}[b] \oplus\left(x_{1}^{j}[b+1] \oplus 1\right) x_{1}^{j}[b+2] \oplus x_{1}^{j}[b+1] x_{2}^{j}[b+2] \oplus x_{2}^{j}[b+1] x_{1}^{j}[b+2]$ for $j>0$
$X_{3}^{j}[b] \leftarrow x_{2}^{j}[b] \oplus\left(x_{2}^{j}[b+1] \oplus 1\right) x_{2}^{j}[b+2] \oplus x_{2}^{j}[b+1] x_{3}^{j}[b+2] \oplus x_{3}^{j}[b+1] x_{2}^{j}[b+2]$ for $j>0$

For $b=3,4$ :

$X_{1}^{j}[b] \leftarrow x_{3}^{j}[b] \oplus\left(x_{3}^{j}[b+1] \oplus 1\right) x_{3}^{j}[b+2] \oplus x_{3}^{j}[b+1] x_{1}^{j}[b+2] \oplus x_{1}^{j}[b+1] x_{3}^{j}[b+2] \oplus x_{3}^{j-1}[b]$ for $j>0$

$X_{2}^{j}[b] \leftarrow x_{1}^{j}[b] \oplus\left(x_{1}^{j}[b+1] \oplus 1\right) x_{1}^{j}[b+2] \oplus x_{1}^{j}[b+1] x_{2}^{j}[b+2] \oplus x_{2}^{j}[b+1] x_{1}^{j}[b+2] \oplus x_{2}^{j-1}[b]$ for $j>0$

$X_{3}^{j}[b] \leftarrow x_{2}^{j}[b] \oplus\left(x_{2}^{j}[b+1] \oplus 1\right) x_{2}^{j}[b+2] \oplus x_{2}^{j}[b+1] x_{3}^{j}[b+2] \oplus x_{3}^{j}[b+1] x_{2}^{j}[b+2] \oplus x_{2}^{j-1}[b] \oplus x_{3}^{j-1}[b]$ for $j>0$

$X_{2}^{0}[b]=C G_{2}[b-3]$

$X_{3}^{0}[b]=C G_{3}[b-3]$

The S-Box index $j$ assumes a one-dimensional array extracted from the three-dimensional state of KeCCAK (Fig. 1). For each plane in the abscissa axis (x) of the state, an array is derived by unrolling the plane, where $0<j \leq y+5 z$.

three times less cycles than the PARALLEL double clocked and the PARALLEL pipelined implementations from [18]. On the other hand, our implementation is between $6 \%$ to $28 \%$ (depending on the library) larger in area than the previous works.

\section{B. Monkeyduplex}

MONKEyDuPLEX implements three functions in its framework: $D$.start, $D$.step and $D$.stride. All of them call the same permutation, but for different numbers of rounds: $n_{\text {start }}=12$, $n_{\text {step }}=1, n_{\text {stride }}=6$. The first one gets as inputs a string forged with the Key and the Nonce, while the others get a block $\sigma$ from either the Associated Data or the Plaintext. Only one KECCAK- $p *$ block is instantiated, which means that the input string and the number of rounds are decided based on the function to run. The three aforementioned functions are encoded in a two bit control signal mode as: 00,10 and 11 respectively.

Two padding blocks are instantiated, one for D.start operation and another one for D.step and D.stride that append a string with the following structure: $10 \ldots 01$ with enough zeros to get the required length. Fig. 7 depicts the diagram of this block: the unprotected version is represented by the black datapath, and the protected version by the red dotted one.

\section{MONKEyWrap}

The last block of the building process is MONKEYWRAP, which instantiates a single MONKEyDuPlex block. The function call can be selected with the mode signal, which is decided by a Control module. This module receives two inputs that indicate when the last block of the Associated Data and the last block of the Plaintext are input. Together with a counter, the Control module decides the value of mode signal, when to load, what to input, and when to enable MonkEYDuPLEX. The length of the input $\sigma$ may vary depending on the absorbed block. Since in the hardware we can not instantiate a bus to variable number of bits (e.g., first 16 bits and then 20 bits), we

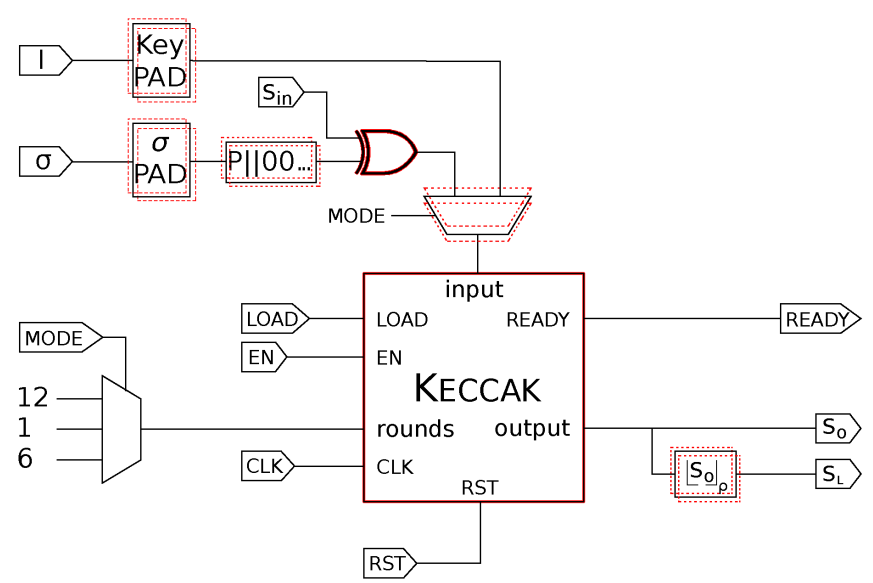

Fig. 7: Unprotected and protected MonKeyDuPLEX implementation

declare the bus width driving $\sigma$ with a fixed maximal length and an integer $\sigma_{l n}$ is attached to it, indicating the actual length of the bus. Thus, we can correctly apply the corresponding padding. Depending on the encryption stage, when computing the associated data, the plaintext, or the tag, MonkEYDUPLEX receives as input $A_{i}\left(\left|A_{i}\right|\right.$ appended), $B_{i}$ (|$\left|B_{i}\right|$ appended), or 0 ( 1 appended) respectively.

The output of MONKEYDUPLEX is then XORed with the next input plaintext block to get the ciphertext, which is output only while the plaintext is being absorbed.

Very few extra hardware is needed to extend the encryption block to support decryption as well. We add a general control signal decrypt, which decides if the block encrypts or decrypts. New control logic is needed to decide the input to MonkeyDuplex, between the input plaintext and the just decrypted ciphertext, after the associated data is fully processed. In order to have the same states as in the encryption procedure, we need to absorb the plaintext, so that is why we first get 
the plaintext block and then feed it into MONKEYDUPLEX to get the next state. According to the specifications, the decrypted plaintext is released all at once. This is only possible once the new tag $T^{\prime}$ is calculated and then checked that it matches with the received one $T_{i n}^{\prime}$. Thus, we implement the tags comparison and optionally add new registers to keep the already calculated plaintext while the new tag is calculated. For very long messages, the total size of these registers might become an impractical area overhead, so one can decide to omit them and output the plaintext while being computed. Fig. 8 illustrates the schematic of MONKEYWRAP, where the unprotected encryption is represented in solid line, and the extension to allow decryption in dashed line.

\section{Securing the implementations}

To protect these last two blocks is easier than the underlying permutation since only linear operations are used. The red datapth in Fig. 7 represents the protected implementation of MonkeyDuplex. We instantiate the secure KECCAK- $p$ * (detailed in IV-A0b), and to secure the linear modules, simple repetition suffices. Note that for the padding we also instantiate it three times; however, this would not work in the case of a design with even shares because the padded string would be canceled when unsharing. In general, it is enough (mandatory in the case of even number of shares) to apply the padding to just one share and pad the rest of the shares with all zeros. The same procedure applies when securing a constant addition.

We follow the same procedure to secure MONKEYWRAP: the protected MONKEYDUPLEX is instantiated, and the surrounding logic including Muxes, paddings, the shift register, and the comparator are repeated three times, one per each share.

The control signals do not carry sensitive information, hence we do not need to protect them nor protect the Control block. When securing the decryption datapath, a problem arises. How do we compare the new calculated tag shared with the input tag? To do the comparison we have to unshare the calculated $T^{\prime}$ as both tags are most certainly shared with different random masks. Reconstructing the tag from the shares would result in a non-completeness violation, where a single wire (probe) would carry information of all shares. This does not entail a problem since the attacker already knows the tag, which is given as input to the decryption. Probing, and getting a different tag, just means that the attacker learns something went wrong in the authentication process, but he never learns secret data.

\section{Results}

Here we present the synthesis results for all the implementations: area, latency, max. frequency, and power. Other than the four bits appended to the state to act as "guards", which are given together with the initial sharing, there is no extra randomness needed. Tab. II summarizes them.

Three shares are used to secure our implementations. Intuitively, the protected implementations should be three times larger in area than the unprotected ones. Instead, they are around 3.6 times larger. The extra overhead comes from the more complex implementation of the non-linear function.

For the encryption\&decryption implementation results, we report the version where the decrypted plaintext is released on the fly as the main architecture. Note that the area increase of the encryption\&decryption scheme is minimal compared to the just-encryption module. Additionally, as an optional choice and to comply with the specifications, we provide the results for the implementation where the plaintext is not revealed until the tags are verified (included in parenthesis in Tab. II). We implement a shift register that stores the decrypted plaintext block by block. To be able to decrypt any message in the session, the shift register has to be the size of the longest ciphertext message. This means the area of the decryption depends on the length of the ciphertext/plaintext. The area of the shift register is given in function of the number of plaintext bits $\# B$, where the expression has two coefficients: the first one refers to the combinational area per bit, calculated experimentally, and the second one refers to the sequential area, 5.67 GE being the area of a single bit register.

We use a special notation to indicate the latency in the KETJE implementations: Cycles per block for processing the Associated Data + Cycles per block for the Plaintext +6 (stride cycles) + \#cycles to produce 128 bit tag. We do not include here the cycles needed to initialize a session, which are always 12 cycles. In encryption mode the Ciphertext is available after Associated Data and Plaintext have been processed. In decryption mode both Plaintext and Tag are given at the end Note that smaller versions take longer to process since the absorption rate is smaller.

The results gathered in Tab. II are given using the NanGate 45nm Open Cell Library [19] and synthesized with Synopsys DC Compiler 2017.09. We compile with -exact_map option, and medium effort for mapping, area, and power to minimize the differences between the written and the synthesized code, and to avoid optimizations that could affect the security.

\section{EVALUATION}

To test our implementations in practice, we deploy them into FPGA and conduct a leakage detection experiment. No information leakage is detected with up to 100 million traces.

1) TVLA: We use the method presented in [20], known as non-specific test vector leakage assessment (TVLA) to detect leakage, or similarly, to detect if intermediate variables have correlation with secret data. This test is not an attack, and retrieving the key is not the aim. This test gives information about the leakage, whose presence is a necessary condition, but not sufficient, for an attack to succeed. The fact that no leakage is observed, gives confidence to the designer on the security of the design. A Differential Power Analysis (DPA) based evaluation of another Authenticated Encryption scheme can be found in [21].

We mount the experiment following the methodology described in [22]. We collect power traces from two different groups (one fixed and the other one random) and compare the two sets using the t-test statistic. When the t-statistic surpasses 


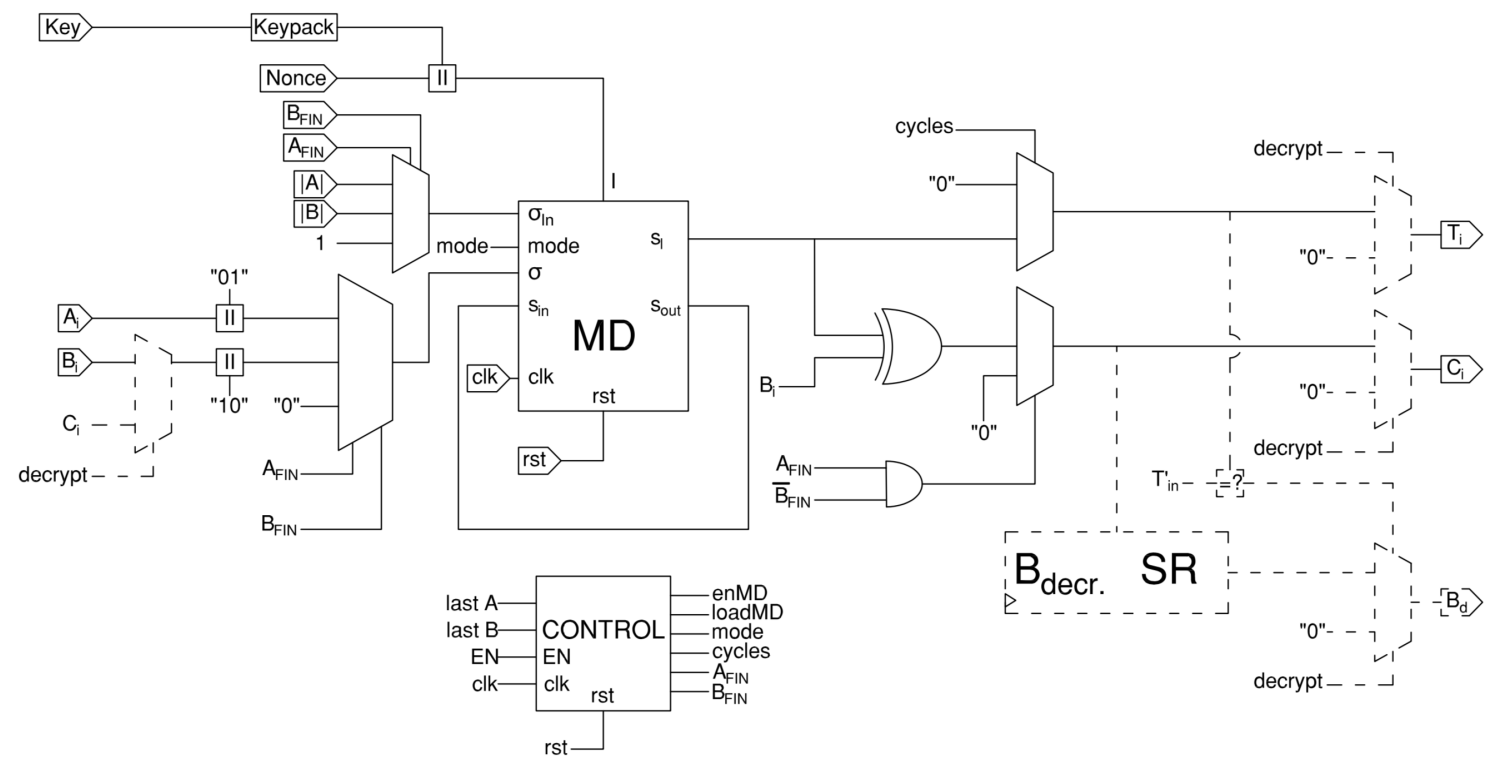

Fig. 8: The MONKEYWRAP implementation including encryption\&decryption

TABLE II: Synthesis results for the entire KeTJE family using the NanGate 45nm Open Cell Library

\begin{tabular}{|c|c|c|c|c|c|c|}
\hline \multirow[t]{2}{*}{ Design } & \multirow{2}{*}{$\begin{array}{l}\text { State } \\
\text { (bits) }\end{array}$} & \multicolumn{2}{|r|}{ Area(GE) } & \multirow{2}{*}{$\begin{array}{c}\text { Latency } \\
\text { (per round/block) }\end{array}$} & \multirow{2}{*}{$\begin{array}{c}\text { Max.Freq. } \\
\text { (MHz) }\end{array}$} & \multirow{2}{*}{$\begin{array}{l}\text { Power } \\
(\mathrm{mW})\end{array}$} \\
\hline & & $\chi$ & Total & & & \\
\hline \multicolumn{7}{|l|}{ Protected KECCAK } \\
\hline$p *[200]$ & 200 & 6319 & 15113 & 1 & 892.85 & 1.58 \\
\hline$p *[400]$ & 400 & 12639 & 30104 & 1 & 892.85 & 2.87 \\
\hline$p *[800]$ & 800 & 25279 & 60092 & 1 & 909.1 & 5.47 \\
\hline$p *[1600]$ & 1600 & 50346 & 119816 & 1 & 877.2 & 9.96 \\
\hline \multicolumn{7}{|c|}{ Unprotected KETJE encryption } \\
\hline$J_{R}$ & 200 & 707 & 5447 & $1+1+6+8$ & 632.9 & 0.682 \\
\hline $\mathrm{SR}$ & 400 & 1413 & 9663 & $1+1+6+4$ & 591.7 & 1.12 \\
\hline MINOR & 800 & 2827 & 19665 & $1+1+6+1$ & 585 & 2.34 \\
\hline MAJOR & 1600 & 5653 & 37650 & $1+1+6+1$ & 543.5 & 4.13 \\
\hline \multicolumn{7}{|c|}{ Protected KETJE encryption } \\
\hline $\mathrm{J}_{\mathrm{R}}$ & 200 & 6319 & 18335 & $1+1+6+8$ & 892.85 & 2.08 \\
\hline $\mathrm{SR}$ & 400 & 12639 & 35136 & $1+1+6+4$ & 892.85 & 3.63 \\
\hline MINOR & 800 & 25279 & 73516 & $1+1+6+1$ & 909.1 & 7.75 \\
\hline MAJOR & 1600 & 50346 & 144022 & $1+1+6+1$ & 877.2 & 14 \\
\hline \multicolumn{7}{|c|}{ Unprotected KETJE encryption\&decryption } \\
\hline$J_{R}$ & 200 & 707 & $6109(+3.6 \cdot \# B+5.67 \cdot \# B)$ & $1+1+6+8$ & 632.9 & 0.93 \\
\hline $\mathrm{SR}$ & 400 & 1413 & $10276(+5.6 \cdot \# B+5.67 \cdot \# B)$ & $1+1+6+4$ & 591.7 & 1.29 \\
\hline MINOR & 800 & 2,827 & $20554(+19.6 \cdot \# B+5.67 \cdot \# B)$ & $1+1+6+1$ & 585 & 2.7 \\
\hline MAJOR & 1600 & 5653 & $39596(+40 \cdot \# B+5.67 \cdot \# B)$ & $1+1+6+1$ & 543.5 & 4.86 \\
\hline \multicolumn{7}{|c|}{ Protected KETJE encryption\&decryption } \\
\hline $\mathrm{JR}_{\mathrm{R}}$ & 200 & 6319 & $20032(+3 \cdot 3.6 \cdot \# B+3 \cdot 5.67 \cdot \# B)$ & $1+1+6+8$ & 892.85 & 2.8 \\
\hline SR & 400 & 12639 & $36645(+3 \cdot 5.63 \cdot \# B+3 \cdot 5.67 \cdot \# B)$ & $1+1+6+4$ & 892.85 & 4,24 \\
\hline MINOR & 800 & 25279 & $77608(+3 \cdot 19.6 \cdot \# B+3 \cdot 5.67 \cdot \# B)$ & $1+1+6+1$ & 909.1 & 9.33 \\
\hline MAJOR & 1600 & 50346 & $145259(+3 \cdot 40 \cdot \# B+3 \cdot 5.67 \cdot \# B)$ & $1+1+6+1$ & 877.2 & 16.4 \\
\hline \multicolumn{7}{|c|}{ Related work [18] } \\
\hline Protected KECCAK & & & & & & \\
\hline PARALlel pip.* & 1600 & 57600 & 111800 & 3 & 837.5 & - \\
\hline PARALLEL d.c.* & 1600 & 44200 & 100500 & 2 & 803.9 & - \\
\hline PARALLEL pip.** & 1600 & 50400 & 97700 & 3 & 846.7 & - \\
\hline PARALLEL d.c.** & 1600 & 38400 & 85700 & 2 & 877.2 & - \\
\hline
\end{tabular}


the threshold of \pm 4.5 , one can conclude with confidence $99.9995 \%$ that the two sets of power traces follow a different distribution and hence, the design leaks.

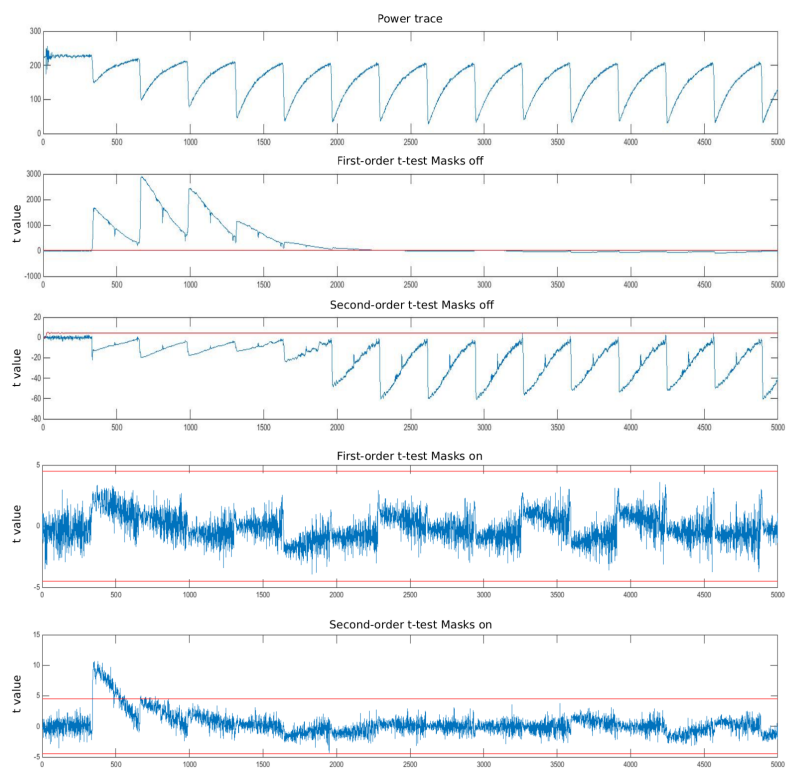

Fig. 9: Welch t-test evaluation of full KECCAK. Top to bottom: power trace, first- and second-order masks off test, and firstand second-order masks on test

2) Setup: To evaluate the security of our implementations we use a SAKURA-G board, specifically designed for sidechannel evaluation, which includes two Spartan-6 FPGAs. To reduce the experimental noise, we split our implementation in the two FPGAs: the control FPGA, which handles the communication with the host computer and generates the shares for the cryptographic FPGA. In the crypto FPGA we deploy the actual scheme. This way we isolate the power consumption of the actual encryption, reducing considerably the noise. We use a very slow $3 \mathrm{MHz}$ clock to ensure clear power traces with minimal overlap between consecutive time samples. The synthesis of the design is done using the Xilinx 14.7 tools with the KEEP HIERARCHY constraint, in order to avoid optimizations across different shares. We measure the power consumption using a Tektronix DPO 7254C oscilloscope, sampling at $1 \mathrm{GS} / \mathrm{s}$ with 5000 points per frame, where fourteen and a half rounds can be appreciated.

\section{A. KECCAK evaluation}

From all the designs, we deploy the smallest KECCAK to test, where $b=200$. Given the properties of the permutation, the conclusions on a specific version of KECCAK transfer to versions of different width. This property is known as the Matryoshka principle [11]. Since KECCAK does not have a key or plaintext as inputs like a regular cipher, we treat the whole input state as secret data. In the evaluation we perform, this translates in the first group being computed with a fixed input, while the second one is computed with a random input that changes for every computation.
We first test the unprotected implementation for 20k traces to verify that our setup is sound and we can find leakage, which is expected in every order. We then activate the countermeasure and run the test again. Now only second-order leakage is expected, since our design has first-order protection. Fig. 9 illustrates this evaluation results. Threshold is represented by the red lines.

\section{B. KeTJE evaluation}

To complete the full KETJE implementation, only linear operations are used on top of KECCAK. As shown in Sec. III-C, linear operations prior to non-linear operations might introduce dependencies among shares and hence noncompleteness failures. Based on the conclusions of that section, we can assume that the KETJE implementation is secure as well, since we use TI to protect it. In the following, we present the practical experiments for the KETJE encryption implementation, confirming our assumptions, and showing that our implementation is secure with up to 100 million traces.

KETJE does have key and plaintext as inputs, so this time we consider solely the key as secret input. We similarly split the computation over two groups, but here both groups receive the same fixed key. Then, both plaintext and associated data are fixed for the first group, while for the second group, they are all random and different in every computation. Thus, we want to see that for the same secret data (key) the computation over the fixed group behaves as random, without revealing any information on the key. Fig. 10 presents the practical evaluation for the complete implementation. Following the same principle as before, we first switch off the countermeasure ("masks off"), to see that the implementation leaks already from first-order as expected. Finally, we switch on the countermeasure and confirm that there is no leakage to be seen in any order.

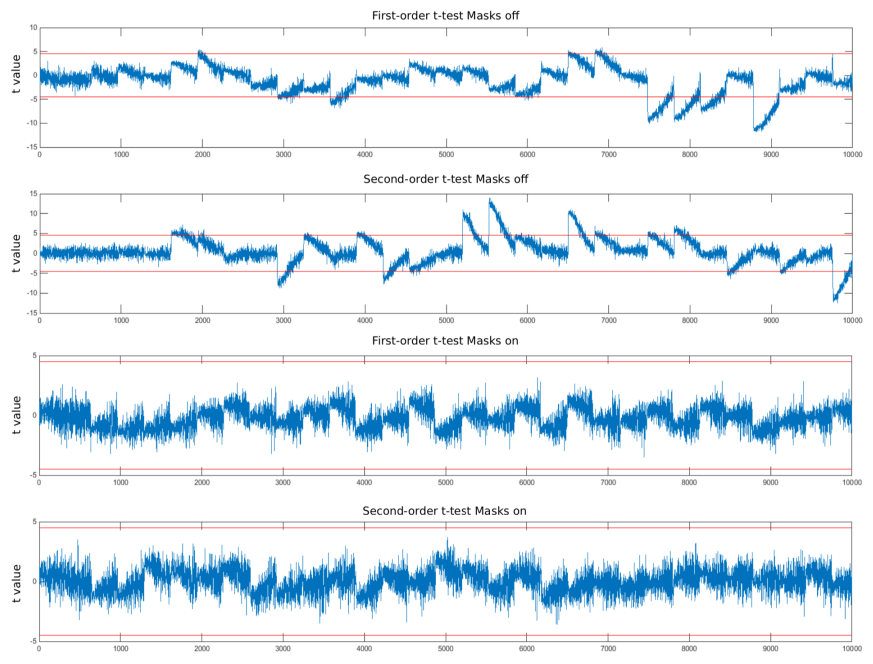

Fig. 10: Welch t-test evaluation of full KETJE encryption. Top to bottom: first- and second-order masks off test, and first- and second-order masks on test

There are several interesting observations to note in this experiments. The first observation is that the leakage in the 
"masks off" experiment appears much later than usual. The plot of this experiment is for 1 million traces, compared to $20 \mathrm{k}$ traces in the KECCAK experiment. Thus, we can empirically confirm that KETJE provides limited SCA resistance, as stated in [10], only up to certain number of computations where the same key is reused. In real applications, the same key should never be used for a significant number of computations. In our experiments we eventually find leakage, since the key is never changed.

The next important observation is that no second-order leakage appears despite our implementation is only first-order secure. Given the small resistance that we observe in the KETJE encryption itself, by introducing the actual side-channel protection on top, we get that leakage does not even appear in the second-order evaluation. Following the same reasoning as before, we presume that leakage will be detected in the second order with enough traces.

\section{CONCLUSION}

In this work we have presented a new first-order protected KECCAK implementation using TI and the "Changing of the Guards" technique. In addition to this, we introduce a general implementation of first-order side-channel protected KETJE authenticated encryption schemes that are suitable for different applications, from lightweight to high throughput purposes. Furthermore, we extend this implementations to include both encryption and decryption functionalities in the same module.

We tested the underlying permutation finding no leakage with up to 100 million traces. By using Threshold Implementations, we are able to transport the security conclusions from the permutation to the whole scheme. Moreover, by implementing the trick of "Changing of the Guards", no extra randomness is needed to provide first-order security, and thus, we do not need to place any dedicated randomness source next to our design, avoiding any problems this might entail.

Finally, we present a TVLA evaluation of the complete KETJE encryption scheme, to successfully verify the initial claim on the security of this implementation with up to 100 million observations. Furthermore, we have seen in our experiments that KETJE by itself provides certain SCA protection, when only a limited number of computations reusing the same key are performed.

\section{ACKNOWLEDGMENT}

This work was partially supported in part by the NIST Research Grant 60NANB15D346, and by the Research Council KU Leuven: C16/18/004.

\section{REFERENCES}

[1] P. C. Kocher, J. Jaffe, and B. Jun, "Differential Power Analysis," in Advances in Cryptology - CRYPTO '99, 19th Annual International Cryptology Conference, Santa Barbara, California, USA, August 15-19, 1999, Proceedings, 1999, pp. 388-397. [Online]. Available: https://doi.org/10.1007/3-540-48405-1_25

[2] S. Mangard, E. Oswald, and T. Popp, Power analysis attacks - revealing the secrets of smart cards. Springer, 2007.
[3] Y. Ishai, A. Sahai, and D. A. Wagner, "Private circuits: Securing hardware against probing attacks," in Advances in Cryptology - CRYPTO 2003, 23rd Annual International Cryptology Conference, Santa Barbara, California, USA, August 17-21, 2003, Proceedings, 2003, pp. 463-481. [Online]. Available: https://doi.org/10.1007/978-3-540-45146-4_27

[4] E. Trichina, "Combinational Logic Design for AES SubByte Transformation on Masked Data," Cryptology ePrint Archive, Report 2003/236, 2003. [Online]. Available: http://eprint.iacr.org/2003/236

[5] S. Nikova, C. Rechberger, and V. Rijmen, "Threshold implementations against side-channel attacks and glitches," In ICICS, volume 4307 of LNCS, pages 529-545. Springer, 2006.

[6] B. Bilgin, B. Gierlichs, S. Nikova, V. Nikov, and V. Rijmen, "Higher-order threshold implementations," In ASIACRYPT, volume 8874 of LNCS, pages 326-343. Springer, 2014.

[7] O. Reparaz, B. Bilgin, S. Nikova, B. Gierlichs, and I. Verbauwhede, "Consolidating masking schemes," in Advances in Cryptology - CRYPTO 2015 - 35th Annual Cryptology Conference, Santa Barbara, CA, USA, August 16-20, 2015, Proceedings, Part I, 2015, pp. 764-783. [Online]. Available: https://doi.org/10.1007/978-3-662-47989-6_37

[8] H. Gross, S. Mangard, and T. Korak, "Domain-Oriented Masking: Compact Masked Hardware Implementations with Arbitrary Protection Order," Cryptology ePrint Archive, Report 2016/486, 2016, http://eprint. iacr.org/2016/486.

[9] V. Arribas, S. Nikova, and V. Rijmen, "Guards in action: First-order SCA secure implementations of ketje without additional randomness," in $21 s t$ Euromicro Conference on Digital System Design, DSD 2018, Prague, Czech Republic, August 29-31, 2018, 2018, pp. 492-499. [Online]. Available: http://doi.ieeecomputersociety.org/10.1109/DSD.2018.00088

[10] G. Bertoni, J. Daemen, M. Peeters, G. V. Assche, and R. V. Keer, "Caesar submission: Ketje v2," September 2016.

[11] G. Bertoni, J. Daemen, M. Peeters, and G. V. Assche, "The keccak reference," http://http://keccak.noekeon.org/, January 2010.

[12] V. Arribas, B. Bilgin, G. Petrides, S. Nikova, and V. Rijmen, "Rhythmic Keccak: SCA security and low latency in hw" IACR Transactions on Cryptographic Hardware and Embedded Systems, vol. 2018, no. 1, pp. 269-290, 2018. [Online]. Available: https: //tches.iacr.org/index.php/TCHES/article/view/840

[13] J. Daemen, "Changing of the guards: A simple and efficient method for achieving uniformity in threshold sharing," in Cryptographic Hardware and Embedded Systems - CHES 2017, W. Fischer and N. Homma, Eds. Cham: Springer International Publishing, 2017, pp. 137-153.

[14] N. Samwel and J. Daemen, "Dpa on hardware implementations of ascon and keyak," in Proceedings of the Computing Frontiers Conference, ser. CF'17. New York, NY, USA: ACM, 2017, pp. 415-424. [Online]. Available: http://doi.acm.org/10.1145/3075564.3079067

[15] F. Wegener and A. Moradi, "A first-order SCA resistant AES without fresh randomness," in Constructive Side-Channel Analysis and Secure Design - 9th International Workshop, COSADE 2018, Singapore, April 23-24, 2018, Proceedings, 2018, pp. 245-262.

[16] G. Bertoni, J. Daemen, M. Peeters, and G. V. Assche, "Building power analysis resistant implementations of Keccak," Second SHA-3 candidate conference, August 2010.

[17] B. Bilgin, J. Daemen, V. Nikov, S. Nikova, V. Rijmen, and G. V. Assche, "Efficient and first-order dpa resistant implementations of keccak," in CARDIS, volume 8419 of LNCS pp 187-199, June 2014

[18] H. Gross, D. Schaffenrath, and S. Mangard, "Higher-order side-channel protected implementations of keccak," Cryptology ePrint Archive, Report 2017/395, 2017, https://eprint.iacr.org/2017/395.

[19] NANGATE, "The NanGate 45nm Open Cell Library," available at http: //www.nangate.com.

[20] J. Cooper, E. D. Mulder, G. Goodwill, J. Jaffe, G. Kenworthy, and P. Rohatgi, "Test Vector Leakage Assessment (TVLA) methodology in practice," International Cryptographic Module Conference, 2013, http://icmc-2013. org/wp/wp-content/uploads/2013/09/goodwillkenworthtestvector.pdf.

[21] H. Groß, E. Wenger, C. Dobraunig, and C. Ehrenhöfer, "Ascon hardware implementations and side-channel evaluation," Microprocessors and Microsystems - Embedded Hardware Design, vol. 52, pp. 470-479, 2017. [Online]. Available: https://doi.org/10.1016/j.micpro.2016.10.006

[22] O. R. B. Gierlichs and I. Verbauwhede, "Fast leakage assessment," in Cryptographic Hardware and Embedded Systems - CHES 2017, W. Fischer and N. Homma, Eds. Cham: Springer International Publishing, 2017, pp. 387-399. 